\title{
Commuting quantum matrix models
}

\section{Veselin G. Filev and Denjoe O'Connor}

School of Theoretical Physics, Dublin Institute for Advanced Studies, 10 Burlington Road, Dublin 4, Ireland

E-mail: vfilev@stp.dias.ie, denjoe@stp.dias.ie

ABSTRACT: We study a quantum system of $p$ commuting matrices and find that such a quantum system requires an explicit curvature dependent potential in its Lagrangian for the system to have a finite energy ground state. In contrast it is possible to avoid such curvature dependence in the Hamiltonian. We study the eigenvalue distribution for such systems in the large matrix size limit. A critical rôle is played by $p=4$. For $p \geq 4$ the competition between eigenvalue repulsion and the attractive potential forces the eigenvalues to form a sharp spherical shell.

KeYwORDS: Matrix Models, 1/N Expansion

ARXiv EPRINT: 1408.1388 


\section{Contents}

1 Introduction 1

2 One matrix quantum model 3

$\begin{array}{lll}2.1 & \text { Gaussian potential } & 7\end{array}$

3 Commuting matrix model $r$

3.1 Gaussian potential 11

4 Conclusions 13

A Scalar curvature of the space of commuting hermitian matrices

\section{Introduction}

Matrix quantum mechanics began [1] with the problem of counting planar diagrams in field theory. In ref. [1] a single matrix was quantised and shown to be equivalent to a free Fermi gas. In the large $N$ (matrix size) limit the eigenvalue density was obtained and found to have similar features to pure matrix models. The eigenvalues are confined to finite domains such that $E_{\mathrm{F}}-V(\lambda)$ is positive and the eigenvalue density is given by $\rho(\lambda)=\sqrt{2 E_{\mathrm{F}}-2 V(\lambda)}$ in these domains with the Fermi energy $E_{\mathrm{F}}$ determined from the normalisation of the eigenvalue density. For a quartic potential the model undergoes a one-cut two-cut phase transition [2] when the quadratic term is made sufficiently negative and other features are broadly in line with the pure matrix model.

Interest in matrix quantum mechanics blossomed with the advent of the AdS/CFT correspondence and multi-matrix models are fundamental to current understanding of Mtheory.

Although a non-perturbative formulation of M-theory in terms of its fundamental degrees of freedom is still lacking, the best candidate for such a formulation appears to be the infinite matrix size limit of a matrix model of some kind. The leading candidate for such a formulation is the BFSS model $[3,4]$ which was conjectured to capture the entire dynamics of M-theory. Relatives of this model such as the BMN model [5] or models derived from the ABJM model $[6,7]$ are also considered possible viable candidates for such a non-perturbative formulation. All of these conjectured formulations of M-theory are regularised versions of the supermembrane and are matrix quantum mechanical systems. They are based on the matrix regularisation of membranes introduced by Hoppe [8] and extended to the supermembrane in [9]. They also arise as dimensional reductions of 4dimensional or 3-dimensional supersymmetric field theories. 
Many of these models will have regimes where commuting matrices play a rôle. It has been suggested by Berenstein [10] that, in order to count 1/8-BPS states, a matrix model of commuting matrices is needed.

Aside from the work of Berenstein [10] there is little known about commutative quantum matrix models. Our goal in this article is to understand the quantisation and quantum mechanics of a system of commuting matrices.

Our first observation is that the configuration space of such matrix models is curved. The consequence of this is that there is no a priori unique quantisation of the system. This is a classic problem when the configuration space is curved and was clarified by B. De Witt in his seminal article [11]. There De Witt found that, if one considers a classical dynamical system in a curved configuration space, the corresponding quantum Hamiltonian has an additional potential given by $\frac{\hbar^{2}}{6} \mathcal{R}$, where $\mathcal{R}$ is the scalar curvature of the configuration space. ${ }^{1}$ Other values for the coefficient of the scalar curvature have been proposed $[11,13]$ but since it is the quantum system that is primary the coefficient can in principal have any value.

For the system of commuting matrices we therefore investigate the general case and allow the coefficient have any value and comment on the different possibilities.

We begin our paper with a fresh derivation of the standard results of matrix quantum mechanics. Using a WKB approximation for the single particle wavefunctions we obtain a direct derivation of the eigenvalue density. We then establish the equivalence of the Bosonic and Fermionic quantisations for the Gaussian potential. We are then in a position to discuss the higher dimensional case where Bosonic quantisation is the relevant one.

The principal results of this paper are:

- The configuration space of $p$-commuting Hermitian matrices with $p>1$ is curved. We compute the curvature of this space and establish that it has a curvature singularity when two eigenvalues approach. This singularity has a negative scalar curvature for $p>1$ independent of $N$.

- If no explicit curvature dependence is included in the action for the system then a path integral quantisation of the system gives rise to a curvature term in the Hamiltonian. We find the resulting system has no finite energy ground state.

- If the Hamiltonian is taken as primary and no explicit curvature dependence is included in this Hamiltonian then for Gaussian commuting matrices we find

(a) For $p=2$ the eigenvalue distribution is uniform within a disc of unit radius.

(b) For $p=3$ the eigenvalues are confined to a ball but have divergent density as the boundary is approached, though the distribution is integrable being $\rho_{3}(r)=\frac{3}{2 \pi^{2} \sqrt{\frac{2}{3}-\vec{r}^{2}}}$.

(c) For $p \geq 4$ competition between eigenvalue repulsion and the attractive potential forces the eigenvalues to form a shell with eigenvalue distribution $\rho_{p}(\vec{x})=$

\footnotetext{
${ }^{1}$ For a more recent discussion see [12].
} 
$\frac{2^{p / 2}}{\Omega_{p-1}} \delta\left(1 / 2-\vec{x}^{2}\right)$ where $\Omega_{p-1}$ is the volume of the unit $p-1$-sphere. Note the critical rôle played by the $p=4$ case.

- We find that if we quantize the $p$-commuting Hermitian matrices with $\frac{\xi}{6} \mathcal{R}$ as the scalar curvature contribution to the Hamiltonian then for $\xi \geq 1$ the system has no finite energy ground state for any polynomial potential. For $\xi<1$ the system is stable and for Gaussian potentials the effect of the curvature term is to scale the radius by a factor of $(1-\xi)^{1 / 4}$. As $\xi \rightarrow 1$ we see the radius goes to zero and the distribution collapses.

As an aside we compute the scalar curvature of a generic squashed flag manifold where the squashing radii are given by the distances between eigenvalues. The scalar curvature of such a flag is always positive. It is the orthogonal complement of the flag in the configuration space of commuting matrices that gives the negative curvature. The space can be envisaged as a warped cone over the flag.

The layout of the paper is as follows: in section two we review the one matrix case giving a novel and explicit derivation of the eigenvalue density. We then establish, in the case of a quadratic potential, the equivalence of the Fermionic and Bosonic quantisations. The Bosonic quantisation will be the one relevant to the subsequent sections. In section three we study commuting matrices observing, to begin with, that the configuration space of such a system is curved. We relegate the derivation of the curvature to appendix A which includes a derivation of the curvature of a generic squashed flag manifold. We then establish, for curvature potential $\frac{\xi}{6} \mathcal{R}$, that when $\xi \geq 1$ the system has no finite energy ground state. In section 3.1 we discuss the range $\xi<1$ and find that for a quadratic confining potential the system is well defined and the eigenvalues are distributed uniformly within a disc for $p=2$, while for $p=3$ they are confined to a finite domain with the density increasing towards the boundary in a divergent but integral form. A special rôle is played by $p=4$ in that it is the lowest dimension in which the eigenvalues are concentrated in a spherical shell. For all $p>4$ the eigenvalues form a spherical shell with the radius specified by $R_{p}^{2}=\sqrt{1-\xi} / 2$ and as $\xi$ approaches one the extent of the eigenvalues goes to zero.

We end the paper with discussion and set the problem in the wider context by relating the system to Calogero-Sutherland-Marchioro models and for $p=2$ to anyonic systems.

\section{One matrix quantum model}

In this section we revisit the quantisation of a one matrix model first presented in [1]. Our goal is to perform a semiclassical quantisation, in the fermionic description of [1]. For the special case of gaussian potential we also demonstrate the equivalence of the bosonic quantisation of the model. It is the bosonic quantisation which admits a natural generalisation to the case with more than one commuting matrix.

Our starting point is the classical Lagrangian:

$$
\mathcal{L}=\operatorname{tr}\left(\frac{1}{2} \dot{X}^{2}-V(X)\right)
$$


where $X$ is an $N \times N$ time dependent Hermitian matrix. The dot represents a time derivate and $V(X)$ is a potential which can be taken to be of the form:

$$
V(X)=\frac{1}{2} X^{2}+\frac{g}{N} X^{4}+\cdots
$$

Hermitian matrices involve complex entries and the space of Hermitian matrices is endowed with the flat metric:

$$
d s^{2}=\operatorname{tr}\left(d X d X^{\dagger}\right)=\operatorname{tr}(d X d X) .
$$

We could proceed and directly quantise the model by using the Hamiltionian [1]:

$$
\begin{aligned}
H & =-\frac{1}{2} \nabla^{2}+V \\
\nabla^{2} & =\sum_{i} \frac{\partial^{2}}{\partial X_{i i}^{2}}+\frac{1}{2} \sum_{i<j} \frac{\partial^{2}}{\partial \operatorname{Re} X_{i j}^{2}}+\frac{\partial^{2}}{\partial \operatorname{Im} X_{i j}^{2}} .
\end{aligned}
$$

But our interest will be in a system of commuting matrices, where the elements of the matrices are not independent and in this case when the constraints are solved we will see that commuting matrices have a curved configuration space. It is therefore most convenient to perform the quantisation in the "curved" coordinates obtained by diagonalising the matrices. These complications are not necessary in the one matrix model, where the configuration space is flat and diagonalisation is just a change of coordinates. However, we will still find it instructive to follow this path in the quantisation of the one matrix model.

Diagonalising the matrix $X$ with a unitary transformation $U$ so that $X=U \Lambda U^{\dagger}$ we arrive at the following expression for the metric (2.3):

$$
d s^{2}=\operatorname{tr}\left(d \Lambda^{2}+[\Lambda, \theta]^{2}\right)=\sum_{i} d \lambda_{i}^{2}+2 \sum_{i<j}\left(\lambda_{i}-\lambda_{j}\right)^{2} \theta_{i j} \bar{\theta}_{i j}
$$

where $\theta=U^{\dagger} d U$ are the Maurer-Cartan left invariant forms and $\lambda_{i}=\Lambda_{i i}$. Furthermore, in these coordinates the Lagrangian (2.1) is given by:

$$
\mathcal{L}=\frac{1}{2} \operatorname{tr}\left(\dot{\Lambda}^{2}+[\Lambda, \dot{\theta}]^{2}\right)-\operatorname{tr} V(\Lambda)=\frac{1}{2} \sum_{i} \dot{\lambda}_{i}^{2}+\sum_{i<j}\left(\lambda_{i}-\lambda_{j}\right)^{2} \dot{\theta}_{i j} \overline{\dot{\theta}}_{i j}-\sum_{i} V\left(\lambda_{i}\right)
$$

where $\dot{\theta}=U^{\dagger} \dot{U}$. Note that the potential is independent on the $\theta$ 's (and $U$ ) and they are cyclic variables. Our next step is to define generalised momenta:

$$
\Pi_{\lambda_{i}}=\frac{\partial \mathcal{L}}{\partial \dot{\lambda}_{i}}=\dot{\lambda}_{i}, \quad \Pi_{\theta_{i j}}=\frac{\partial \mathcal{L}}{\partial \dot{\bar{\theta}}_{i j}}=\left(\lambda_{i}-\lambda_{j}\right)^{2} \dot{\theta}_{i j}, \quad \Pi_{\bar{\theta}_{i j}}=\frac{\partial \mathcal{L}}{\partial \dot{\theta}_{i j}}=\bar{\Pi}_{\theta_{i j}}, \quad \text { for } i<j .
$$

For the corresponding Hamiltonian we obtain:

$$
H=\frac{1}{2} \sum_{i} \Pi_{\lambda_{i}}^{2}+\sum_{i<j} \frac{\Pi_{\theta_{i j}} \Pi_{\bar{\theta}_{i j}}}{\left(\lambda_{i}-\lambda_{j}\right)^{2}}+\sum_{i} V\left(\lambda_{i}\right)=\frac{1}{2} g^{a b} \Pi_{a} \Pi_{b}+\sum_{i} V\left(\lambda_{i}\right),
$$


where $a, b$ are collective indices for the $\lambda_{i}, \theta_{i j}$ and $\bar{\theta}_{i j}$ components and $g^{a b}$ is the inverse of the metric (2.6). The general prescription for quantising the Hamiltonian (2.9) suggest that we construct the operator:

$$
\begin{aligned}
& \hat{H}=-\frac{1}{2} \frac{1}{\sqrt{g}} \partial_{a}\left(\sqrt{g} g^{a b} \partial_{b}\right)+\operatorname{tr} V(\lambda)=-\frac{1}{2 \Delta} \sum_{i} \partial_{\lambda_{i}}\left(\Delta \partial_{\lambda_{i}}\right)-\sum_{i<j} \frac{\mathcal{L}_{\theta_{i j}} \mathcal{L}_{\bar{\theta}_{i j}}}{\left(\lambda_{i}-\lambda_{j}\right)^{2}}+\operatorname{tr} V(\lambda), \\
& \text { where } \Delta=\prod_{i>j}\left(\lambda_{i}-\lambda_{j}\right)^{2} .
\end{aligned}
$$

Note that the differential operators $\mathcal{L}_{\theta_{i j}}$ and $\mathcal{L}_{\bar{\theta}_{i j}}$ are left invariant vector fields dual to the left invariant Maurer-Cartan forms. ${ }^{2}$ The eigenvalues of the operator (2.10) determine the energy of the system. Therefore we need to solve the corresponding Schrödinger equation:

$$
\hat{H} \Psi=N^{2} E^{(1)} \Psi
$$

where we use the notation of ref. [1]. Given that the potential depends only on the eigenvalues of the matrix we consider separate angular and eigenvalue dependence. Furthermore our goal is only the ground state energy which will be given by the angular independent wave function $\Psi\left(\lambda_{1}, \ldots, \lambda_{N}\right)$, which is a function only of the eigenvalues. Following ref. [1] we consider the redefinition:

$$
\phi\left(\lambda_{1}, \ldots, \lambda_{N}\right)=\prod_{i>j}\left(\lambda_{i}-\lambda_{j}\right) \Psi\left(\lambda_{1} \ldots \lambda_{N}\right) .
$$

Note that in order for the original wave function $\Psi\left(\lambda_{1} \ldots \lambda_{N}\right)$ to be analytic as $\lambda_{i} \rightarrow \lambda_{j}$, we need the new wave function $\phi\left(\lambda_{1} \ldots \lambda_{N}\right)$ to be completely antisymmetric, therefore the quantisation of the model is equivalent to the quantisation of $N$ fermions in the central potential $V$. Indeed, the Schrödinger equation for the wave function (2.12) becomes [1]:

$$
\sum_{i}\left(-\frac{1}{2} \frac{\partial^{2}}{\partial \lambda_{i}^{2}}+V\left(\lambda_{i}\right)\right) \phi=N^{2} E^{(1)} \phi .
$$

The antisymmetric wave function $\phi$ can be constructed as the Slater determinant:

$$
\phi\left(\lambda_{1}, \ldots, \lambda_{N}\right)=\frac{1}{\sqrt{N !}} \sum_{i_{1}, \ldots, i_{N}} \varepsilon_{i_{1} \ldots i_{N}} f_{i_{1}}\left(\lambda_{1}\right) \ldots f_{i_{N}}\left(\lambda_{N}\right),
$$

where $f_{i}$ are individual wave functions normalised to one and satisfying:

$$
\left[-\frac{1}{2} \frac{\partial^{2}}{\partial \lambda^{2}}+V(\lambda)\right] f_{i}(\lambda)=e_{i} f_{i}(\lambda)
$$

and $\sum_{i} e_{i}=N^{2} E^{(1)}$, since we are interested in the ground state of the system we choose the individual energies $e_{i}$ to label the first $N$ excited states of the individual hamiltonian.

\footnotetext{
${ }^{2}$ Note also that in general there is the freedom to add to the potential a term proportional to the scalar curvature, which for one matrix vanishes, because the metric (2.6) is flat.
} 
Now the distribution of the eigenvalues can be obtained from the modulus of the wave function $\phi$ by integrating over all but one of the eigenvalues:

$$
\begin{aligned}
\rho(\lambda)=\int \prod_{k=2}^{N} d \lambda_{k}\left|\phi\left(\lambda, \lambda_{2}, \ldots \lambda_{N}\right)\right|^{2} & =\sum_{i_{2}, \ldots, i_{N}} \varepsilon_{i, i_{2} \ldots, i_{N}} \varepsilon_{j i_{2} \cdots N} \frac{f_{i}(\lambda) f_{j}^{*}(\lambda)}{N !} \\
& =\frac{1}{N} \sum_{i}\left|f_{i}(\lambda)\right|^{2} .
\end{aligned}
$$

Therefore, we see that the distribution of the eigenvalues is given as an average over the probability distributions determined by the individual wave functions $f_{i}$. The key idea to obtain a closed form expression for the distribution $\rho$ valid at large $N$, is to notice that at large $N$ the semi-classical approximation to the individual wave function is well justified and in the limit $N \rightarrow \infty$ becomes exact. Indeed, rescaling the eigenvalues, and redefining the wave functions, the potential and the individual energies via:

$$
\lambda_{i} \rightarrow \sqrt{N} \lambda_{i}, \quad \tilde{f}_{i}(\lambda)=N^{1 / 4} f_{i}(\sqrt{N} \lambda), \quad u(\lambda)=\frac{1}{N} V(\sqrt{N} \lambda), \quad \varepsilon_{i}=\frac{e_{i}}{N}
$$

we arrive at:

$$
\left[-\frac{1}{2 N^{2}} \frac{\partial^{2}}{\partial \lambda^{2}}+u(\lambda)\right] \tilde{f}_{i}(\lambda)=\varepsilon_{i} \tilde{f}_{i}(\lambda) .
$$

We see that we have an effective Planck constant $\hbar \propto 1 / N$, and for large $N$ we can use a WKB approximation for the wave functions $[14] \tilde{f}_{i}(\lambda)$ :

$$
\begin{aligned}
\tilde{f}_{i}(\lambda) & =\frac{C_{1}}{\sqrt{p(\lambda)}} \cos \left(N \int_{a}^{\lambda} d \lambda^{\prime} p\left(\lambda^{\prime}\right)-\frac{\pi}{4}\right), \\
p(\lambda) & =\sqrt{2\left(\varepsilon_{i}-u(\lambda)\right)}
\end{aligned}
$$

for $a \leq \lambda \leq b$, where $a$ and $b$ are the two turning points of the classical trajectory. The normalisation coefficient $C_{1}$ is fixed by the condition:

$$
\int_{a}^{b} d \lambda\left|\tilde{f}_{i}(\lambda)\right|^{2} \approx \frac{C_{1}^{2}}{2} \int_{a}^{b} \frac{d \lambda}{p(\lambda)}=\frac{\pi C_{1}^{2}}{2 \omega_{i}}=1,
$$

where $\omega_{i}=2 \pi / T_{i}$ is the frequency of the classical motion depending on the energy $\varepsilon_{i}$, and we have used that for large $N$ to leading order we have $\cos ^{2}\left(N \int^{\lambda} d \lambda^{\prime} p\left(\lambda^{\prime}\right)\right) \approx 1 / 2$ under the integral in (2.21). With this normalisation, and approximating again the fast oscillating cosine function with one half, for the absolute value square of the wave function $\tilde{f}_{i}$ at large $N$ we obtain:

$$
\left|\tilde{f}_{i}(\lambda)\right|^{2}=\frac{\omega_{i}}{\pi \sqrt{2\left(\varepsilon_{i}-u(\lambda)\right)}} .
$$

Redefining the distribution for the rescaled eigenvalues (so that it is normalised to one) via:

$$
\tilde{\rho}(\lambda)=\sqrt{N} \rho(\sqrt{N} \lambda)=\frac{1}{N} \sum_{i}\left|\tilde{f}_{i}(\lambda)\right|^{2},
$$


we obtain:

$$
\tilde{\rho}(\lambda)=\frac{1}{N} \sum_{n} \frac{\omega_{n}}{\pi \sqrt{2\left(\varepsilon_{n}-u(\lambda)\right)}} .
$$

Our final step is to use the Born-Sommerfeld quantisation (for $\hbar=1 / N$ ):

$$
\frac{N}{2 \pi} \oint p d \lambda=n+\frac{1}{2}
$$

to obtain:

$$
\frac{N}{2 \pi} \oint \Delta p_{n} d \lambda=\frac{N \Delta \varepsilon_{n}}{2 \pi} \oint \frac{\partial p_{n}}{\partial \varepsilon_{n}} d \lambda=\frac{N \Delta \varepsilon_{n}}{2 \pi} \oint \frac{d \lambda}{p_{n}}=\frac{N \Delta \varepsilon_{n}}{\omega_{n}}=\Delta n .
$$

The last equality allows us (in the large $N$ limit) to express the sum over $n$ in (2.24) as a definite integral over $\varepsilon$ :

$$
\tilde{\rho}(\lambda)=\sum_{n} \frac{\Delta \varepsilon_{n}}{\pi \sqrt{2\left(\varepsilon_{n}-u(\lambda)\right)}}=\frac{1}{\pi} \int_{u(\lambda)}^{\epsilon_{f}} \frac{d \varepsilon}{\sqrt{2(\varepsilon-u(\lambda))}}=\frac{1}{\pi} \sqrt{2\left(\varepsilon_{f}-u(\lambda)\right)},
$$

which is our final expression for the distribution $\tilde{\rho}$ and agrees with the result in ref. [1], as it should, since we have only made the semiclassical analysis more explicit.

\subsection{Gaussian potential}

Let us now focus on the specific case of a gaussian potential $V(X)=X^{2} / 2$. In this case the rescaled individual wave functions $\tilde{f}_{n}$ satisfy the Schrödinger equation for the one dimensional harmonic oscillator:

$$
\left[-\frac{1}{2 N^{2}} \frac{\partial^{2}}{\partial \lambda^{2}}+\frac{1}{2} \lambda^{2}\right] \tilde{f}_{n}(\lambda)=\varepsilon_{n} \tilde{f}_{n}(\lambda)
$$

where:

$$
\tilde{f}_{n}(\lambda)=\frac{H_{n}(\lambda) e^{-N \lambda^{2} / 2}}{\sqrt{\pi^{1 / 2} 2^{n} n !}} ; \quad \varepsilon_{n}=\frac{(1 / 2+n)}{N} ;
$$

and $H_{n}(\lambda)$ are the Hermite polynomials. The corresponding multi-particle wave function is given by:

$$
\begin{aligned}
\tilde{\phi}\left(\lambda_{1}, \ldots, \lambda_{N}\right) & =C_{N} \sum_{i_{1}, \ldots, i_{N}} H_{i_{1}}\left(\lambda_{1}\right) \ldots H_{i_{N}}\left(\lambda_{N}\right) e^{-N \sum_{i} \lambda_{i}^{2} / 2} \\
& =2^{\frac{N(N-1)}{2}} C_{N} \prod_{i>j}\left(\lambda_{i}-\lambda_{j}\right) e^{-N \sum_{i} \lambda_{i}^{2} / 2}
\end{aligned}
$$

where $C_{N}^{-2}=N ! \prod_{n=0}^{N-1}\left(\pi^{1 / 2} 2^{n} n !\right)$ and we have used the properties of the Hermite polynomials to arrive at the last equality. Equation (2.30) suggests that the bosonic wave function $\tilde{\psi}$ before the change of variables (2.12) is given by:

$$
\tilde{\Psi}\left(\lambda_{1}, \ldots, \lambda_{N}\right)=2^{\frac{N(N-1)}{2}} C_{N} \prod_{i} e^{-N \lambda_{i}^{2} / 2} .
$$


Therefore, the bosonic wave function of the ground state of the system is a product of individual wave functions. We could have easily guessed this result even directly in the bosonic description without introducing the new wave function (2.12). Note that this is true only for the case of a gaussian potential. In fact, in the next section we will show that this holds even for the case of more than one commuting matrices with a gaussian potential [10].

\section{Commuting matrix model}

In this section we focus on the quantisation of the commuting matrix model. While we will set up the problem for general interaction potential the main findings that we present are for the special case of gaussian potential, first discussed in ref. [10]. Our starting point is the lagrangian:

$$
\mathcal{L}=\operatorname{tr}\left(\frac{1}{2} \dot{\vec{X}}^{2}-V(\vec{X})\right)
$$

where $\vec{X}$ represent a set of $p$ commuting $N \times N$ hermitian matrices:

$$
\vec{X}=\left\{X^{1}, \ldots X^{p}\right\} \quad \text { and } \quad\left[X^{\mu}, X^{\nu}\right]=0 \quad \text { for } \quad \mu, \nu=0, \ldots, p .
$$

Unlike the one matrix model discussed in the previous section we cannot directly write down the corresponding hamiltonian. The problem is that the commuting matrices are not independent and the system is constrained. This is why we will perform the quantisation in "curved" coordinates by using the parameterisation:

$$
X^{\mu}=U \Lambda^{\mu} U^{\dagger}, \quad \text { for } \quad \mu=1, \ldots, p,
$$

where $\Lambda^{\mu}$ are real diagonal matrices consisting of the eigenvalues of the commuting matrices $X^{\mu}$ and $U$ is an unitary matrix. Note that $U$ is defined modulo a right multiplication by a diagonal unitary matrix. Taking the quotient with respect to this equivalence leaves only $N^{2}-N$ independent real components of the unitary matrix $U$, which together with $p N$ real degrees of the eigenvalue matrices $\Lambda^{\mu}$ suggests that the space of the $p$ commuting matrices is an $N^{2}+(p-1) N$ dimensional subspace in the $p N^{2}$ space of independent hermitian matrices. Using the natural flat metric in the space of independent hermitian matrices, for the induced metric on the space of commuting matrices we obtain:

$$
d s^{2}=\operatorname{tr}(d \vec{X} \cdot d \vec{X})=\sum_{i} d \vec{\lambda}_{i}^{2}+2 \sum_{i>j}\left(\vec{\lambda}_{i}-\vec{\lambda}_{j}\right)^{2} \theta_{i j} \bar{\theta}_{i j}
$$

where $\theta=U^{\dagger} d U$ are the Maurer-Cartan left invariant forms and $\vec{\lambda}_{i}=\vec{\Lambda}_{i i}$ are the eigenvalues of the commuting matrices. In these coordinates the Lagrangian (3.1) is given by:

$$
\mathcal{L}=\frac{1}{2} \operatorname{tr}\left(\dot{\vec{\Lambda}}^{2}+[\vec{\Lambda}, \dot{\theta}]^{2}\right)-\operatorname{tr} V(\vec{\Lambda})=\frac{1}{2} \sum_{i} \dot{\vec{\lambda}}_{i}^{2}+\sum_{i<j}\left(\vec{\lambda}_{i}-\vec{\lambda}_{j}\right)^{2} \dot{\theta}_{i j} \overline{\dot{\theta}}_{i j}-\sum_{i} V\left(\vec{\lambda}_{i}\right),
$$


where $\dot{\theta}=U^{\dagger} \dot{U}$. Defining the conjugate momenta:

$$
\Pi_{\vec{\lambda}_{i}}=\frac{\partial \mathcal{L}}{\partial \dot{\vec{\lambda}}_{i}}=\dot{\vec{\lambda}}_{i}, \quad \Pi_{\theta_{i j}}=\frac{\partial \mathcal{L}}{\partial \dot{\bar{\theta}}_{i j}}=\left(\vec{\lambda}_{i}-\vec{\lambda}_{j}\right)^{2} \dot{\theta}_{i j}, \quad \Pi_{\bar{\theta}_{i j}}=\frac{\partial \mathcal{L}}{\partial \dot{\theta}_{i j}}=\bar{\Pi}_{\theta_{i j}}, \quad \text { for } \quad i<j .
$$

For the corresponding classical Hamiltonian we obtain:

$$
H=\frac{1}{2} \sum_{i} \Pi_{\vec{\lambda}_{i}}^{2}+\sum_{i<j} \frac{\Pi_{\theta_{i j}} \Pi_{\bar{\theta}_{i j}}}{\left(\vec{\lambda}_{i}-\vec{\lambda}_{j}\right)^{2}}+\sum_{i} V\left(\vec{\lambda}_{i}\right)=\frac{1}{2} g^{a b} \Pi_{a} \Pi_{b}+\sum_{i} V\left(\vec{\lambda}_{i}\right),
$$

where $a, b$ are a collective indices for the $\lambda_{i}^{\mu}, \theta_{i j}$ and $\bar{\theta}_{i j}$ components of the inverse of the metric (3.4). So far the analysis was a simple generalisation of the one performed in the one matrix model case. However, note that unlike the metric (2.6), which is just a flat metric written in curved coordinates, the metric (3.4) is the induced metric on the subspace of commuting matrices and in general there is no reason to expect that it is flat and as it turns out it isn't. It is a well known problem in quantum mechanics in a curved space that there is an ambiguity in the definition of the quantum Hamiltonian. In particular there is the freedom to add a term proportional to the scalar curvature to the potential. The coefficient in front of this term is not determined, however if one demands a path integral quantisation with the naive classical Hamiltonian, this coefficient is fixed to $1 / 6$. Alternatively one could introduce an appropriate such term in the Lagrangian so that the term generated by the measure of the path integral cancels it $[11,12]$ and one ends up with a coefficient zero in the quantum Hamiltonian. It is the quantum system that is more fundamental and since such a term is induced by the path integral measure then, when the system is an effective low energy Lagrangian, it seems natural to allow the coefficient to take on any value. Our strategy will be to keep this coefficient general and explore the implications of varying it. Thus we consider the Hamiltonian.

$$
\begin{aligned}
\hat{H} & =-\frac{1}{2} \frac{1}{\sqrt{g}} \partial_{a}\left(\sqrt{g} g^{a b} \partial_{b}\right)+\operatorname{tr} V(\vec{\Lambda})+\frac{\xi}{6} \mathcal{R} \\
& =-\frac{1}{2 \Delta} \sum_{i} \partial_{\vec{\lambda}_{i}} \cdot\left(\Delta \partial_{\vec{\lambda}_{i}}\right)-\sum_{i<j} \frac{\partial_{\theta_{i j}} \partial_{\bar{\theta}_{i j}}}{\left(\vec{\lambda}_{i}-\vec{\lambda}_{j}\right)^{2}}+\operatorname{tr} V(\vec{\Lambda})+\frac{\xi}{6} \mathcal{R},
\end{aligned}
$$

where $\Delta=\prod_{i>j}\left(\vec{\lambda}_{i}-\vec{\lambda}_{j}\right)^{2}$ and $\mathcal{R}$ is the Ricci scalar curvature of the metric (3.4) for which we obtain the expression:

$$
\begin{aligned}
\mathcal{R} & =-4(p-1) \sum_{i \neq j} \frac{1}{\left(\vec{\lambda}_{i}-\vec{\lambda}_{j}\right)^{2}}-3 \sum_{i \neq j \neq k} \frac{\left(\vec{\lambda}_{i}-\vec{\lambda}_{j}\right)}{\left(\vec{\lambda}_{i}-\vec{\lambda}_{j}\right)^{2}} \cdot \frac{\left(\vec{\lambda}_{i}-\vec{\lambda}_{k}\right)}{\left(\vec{\lambda}_{i}-\vec{\lambda}_{k}\right)^{2}} \\
& =-(4 p+3 N-10) \sum_{i \neq j} \frac{1}{\left(\vec{\lambda}_{i}-\vec{\lambda}_{j}\right)^{2}}+\frac{3}{2} \sum_{i \neq j \neq k} \frac{\left(\vec{\lambda}_{j}-\vec{\lambda}_{k}\right)^{2}}{\left(\vec{\lambda}_{i}-\vec{\lambda}_{j}\right)^{2}\left(\vec{\lambda}_{i}-\vec{\lambda}_{k}\right)^{2}} .
\end{aligned}
$$

As one can see the curvature can be written with negative two body and positive three body interactions, the net effect in the large $N$ limit however is always a negative curvature. A fact which will prove crucial for the quantisation of the model. 
Our next step is to consider a wave function $\Psi$, which is invariant under $\mathrm{SU}(N)$ gauge transformation and thus independent of the $\theta$ 's. The resulting Schrödinger equation has a term involving a first derivative of the wave function, which we can discard by considering the higher dimensional analogue of the change of variables (2.12):

$$
\Phi\left(\vec{\lambda}_{1}, \ldots, \vec{\lambda}_{N}\right)=\prod_{i>j}\left|\vec{\lambda}_{i}-\vec{\lambda}_{j}\right| \Psi\left(\vec{\lambda}_{1}, \ldots, \vec{\lambda}_{N}\right) .
$$

Taking into account equations (3.8), (3.9) and (3.10) for the Schrödinger equation satisfyed by $\Phi$ we obtain:

$$
\begin{aligned}
-\frac{1}{2} \sum_{i} \frac{\partial^{2} \Phi}{\partial \vec{\lambda}_{i}^{2}}+\frac{3-4 \xi}{6}(p-1) \sum_{i \neq j} \frac{\Phi}{\left(\vec{\lambda}_{i}-\vec{\lambda}_{j}\right)^{2}}+\frac{1-\xi}{2} \sum_{i \neq j \neq k} \frac{\left(\vec{\lambda}_{i}-\vec{\lambda}_{j}\right)}{\left(\vec{\lambda}_{i}-\vec{\lambda}_{j}\right)^{2}} \cdot \frac{\left(\vec{\lambda}_{i}-\vec{\lambda}_{k}\right)}{\left(\vec{\lambda}_{i}-\vec{\lambda}_{k}\right)^{2}} \Phi+ & \\
& +\sum_{i} V\left(\vec{\lambda}_{i}\right) \Phi=N^{2} E \Phi
\end{aligned}
$$

Few comments are in order. Notice that the change of variables (3.10) does not affect the symmetry of the wave function and the new wave function $\Phi$ is symmetric thus describing bosons. ${ }^{3}$ This is in contrast to the one dimensional case, where the Vandermonde determinant in (2.12) implied that the new wave function is antisymmetric. Therefore, the problem of quantising the commuting model is equivalent to finding a bosonic wave function satisfying the Schrödinger equation (3.11), which describes $p$-dimensional bosonic particles subjected to the central potential $V$ and with two and three body interaction terms.

Note that for $\xi \geq 1$ the two and three body interactions are attractive, which naively suggests that the model will be unstable [14]. One can of course imagine that the kinetic energy of the particles may stabilise the system. However, as we are going to demonstrate, doing so would require that the number of commuting matrices go to infinity in the large $N$ limit. Indeed, the instability arises, when the eigenvalues are close to each other. In this limit the contribution from the two and tree-body interactions dominate and we can ignore the central potential. Let us first consider the case $\xi$ strictly greater than one, $\xi>1$. Assuming a fixed typical scale for the spread of the eigenvalues $\left|\vec{\lambda}_{i}-\vec{\lambda}_{j}\right| \approx \Delta R$ and using equations (3.11) and (3.9), for the leading order contribution to the potential energy (at large $N$ ) we obtain:

$$
E_{\mathrm{pot}}=-\frac{\xi-1}{2 \Delta R^{2}} N^{3}+O\left(N^{2}\right)
$$

On the other hand, in $p$ dimensions the typical scale along one of the coordinates $\Delta x$ is related to $\Delta R$ via: $\Delta x^{2}=\Delta R^{2} / p$. This suggests that the uncertainty of the momentum along one of the coordinates is $\Delta q \propto \hbar / \Delta x$ and therefore the kinetic energy is of order:

$$
E_{\text {kin }}=N p \Delta q^{2} \propto N p^{2} / \Delta R^{2} .
$$

\footnotetext{
${ }^{3}$ Note that for $p=2$ an alternative quantisation is also possible. One could take the analytic square root of the eigenvalue difference by going to complex coordinates and make contact with anyonic systems [21].
} 
Stabilising the model would require $E_{\text {kin }} \sim\left|E_{\text {pot }}\right|$, comparing equations (3.12) and (3.13) we conclude that this would imply $p \sim N$, and hence in the large $N$ limit the number of matrices should grow proportional to the size of the matrices. For models with a fixed number of commuting matrices $p$, the kinetic energy is not sufficient to stabilise the system for arbitrary large $N$ and the large $N$ limit of these models does not have a ground state for $\xi>1$.

Similar result can be obtained for the case $\xi=1$, when the three body interaction term vanishes. In this case the potential energy is dominated by the two body potential ${ }^{4}$ and to leading order in $N$ one obtains:

$$
E_{\text {pot }}=-(p-1) N^{2} / \Delta R^{2} .
$$

Comparing this expression for the potential energy with the expression for the kinetic energy (3.13), which is still valid, one again arrives at the result that stabilisation of the model in the large $N$ limit requires $p \sim N$ and the commuting matrix model with fixed number of commuting matrices is unstable for $\xi=1$.

Let us demonstrate how this instability occurs in the special case of an attractive gaussian potential.

\subsection{Gaussian potential}

In this subsection we analyse the case of a gaussian potential $V(\lambda)=\frac{1}{2} \lambda^{2}$, first studied in ref. [10] for the case $\xi=0$. For $\xi=0$ one can show that the ground state wave function is given by [10]:

$$
\Phi\left(\vec{\lambda}_{1}, \ldots, \vec{\lambda}_{N}\right)=\prod_{i>j}\left|\vec{\lambda}_{i}-\vec{\lambda}_{j}\right| e^{-\frac{1}{2} \sum_{i} \vec{\lambda}_{i}^{2}}
$$

which is the naive generalisation to higher dimensions of the wave function (2.30). Note that this is an exact result at any $N$. For general $\xi$ it seems that we don't have analytic access to the exact result, however we can still extract the ground state wave function and the corresponding eigenvalue distribution in the large $N$ limit. To see this let us rescale the eigenvalues by: $\vec{\lambda}_{i} \rightarrow N^{1 / 2} \vec{\lambda}_{i}$. After dividing equation (3.11) by $N$ we obtain:

$$
\frac{1}{N} \sum_{i}\left\{-\frac{1}{2 N^{2}} \frac{\partial^{2} \tilde{\Phi}}{\partial \vec{\lambda}_{i}^{2}}+\frac{(3-4 \xi)(p-1)}{6 N^{2}} \sum_{\substack{j \\ j \neq i}} \frac{\tilde{\Phi}}{\left(\vec{\lambda}_{i}-\vec{\lambda}_{j}\right)^{2}}+\frac{1-\xi}{2 N^{2}} \sum_{j \neq k} \frac{\left(\vec{\lambda}_{i}-\vec{\lambda}_{j}\right)}{\left(\vec{\lambda}_{i}-\vec{\lambda}_{j}\right)^{2}} \cdot \frac{\left(\vec{\lambda}_{i}-\vec{\lambda}_{k}\right)}{\left(\vec{\lambda}_{i}-\vec{\lambda}_{k}\right)^{2}} \tilde{\Phi}+\frac{1}{2} \vec{\lambda}_{i}^{2} \tilde{\Phi}\right\}=E \tilde{\Phi} .
$$

Note that the two body interaction (the second) term in equation (3.16) is of order $1 / N$ relative to the other potential terms. Therefore, at least naively it should not contribute to the leading order behaviour of the wave function in the large $N$ limit. With this in mind one can show that the following ansatz for the wave function:

$$
\tilde{\Phi}\left(\vec{\lambda}_{1}, \ldots, \vec{\lambda}_{N}\right)=\prod_{i>j}\left|\vec{\lambda}_{i}-\vec{\lambda}_{j}\right|^{\sqrt{1-\xi}} e^{-\frac{N}{2} \sum_{i} \vec{\lambda}_{i}^{2}},
$$

\footnotetext{
${ }^{4}$ For a discussion of the instability of the attractive $-\frac{\alpha}{x^{2}}$ potential in quantum mechanics with $\alpha>\frac{1}{4}$ see section 35 of [14]. Our argument with the minimum uncertainty $\Delta p=\frac{1}{2 \Delta x}$ reproduces the critical value of $\frac{1}{4}$ for this instability.
} 
satisfies equation (3.16) to leading order in the large $N$ expansion and for the "stabilised" energy $E$ we obtain:

$$
E=\frac{1}{2} \sqrt{1-\xi}+O(1 / N)
$$

To verify the consistency of this approximation we treat the two body interaction term as a perturbation $\delta V$ to the potential of relative order $1 / N$, therefore the corresponding correction to the energy $\delta E$ would also be of order $1 / N$ and we conclude that as long as $E \sim 1$ we can trust the wave function (3.17). Note that this suggests that the parameter $\xi$ is restricted to $\xi<1$, which is consistent with the general arguments (presented in the previous section) that the model is unstable for $\xi \geq 1$. Let us comment on the properties of the eigenvalue distribution for $\xi<1$.

The probability density corresponding to the wave function (3.17) can be written as:

$$
|\tilde{\Phi}|^{2}=\exp \left[-N \sum_{i} \vec{\lambda}_{i}^{2}+\frac{\sqrt{1-\xi}}{2} \sum_{i \neq j} \log \left(\vec{\lambda}_{i}-\vec{\lambda}_{j}\right)^{2}\right] .
$$

The corresponding eigenvalue distribution:

$$
\rho(\vec{\lambda})=\int \prod_{i=2}^{N} d^{p} \lambda_{i}\left|\tilde{\Phi}\left(\vec{\lambda}, \vec{\lambda}_{2}, \ldots, \vec{\lambda}_{N}\right)\right|^{2}
$$

is then equivalent to the eigenvalue distribution of the commuting matrix model described by the action:

$$
S=N \sum_{i} \vec{\lambda}_{i}^{2}-\frac{\sqrt{1-\xi}}{2} \sum_{i \neq j} \log \left(\vec{\lambda}_{i}-\vec{\lambda}_{j}\right)^{2},
$$

which modulo normalisation constants was analysed in refs. [15]-[18]. The corresponding saddle point equation is given by:

$$
\frac{1}{\sqrt{1-\xi}} \vec{\lambda}_{i}=\frac{1}{N} \sum_{j} \frac{\vec{\lambda}_{i}-\vec{\lambda}_{j}}{\left(\vec{\lambda}_{i}-\vec{\lambda}_{j}\right)^{2}}
$$

comparing this to equation (2.6) in ref. [18] we conclude that all of the results of [18] for gaussian potential are valid here provided we multiply the radius of the distribution by a factor of $\left(\frac{1-\xi}{4}\right)^{1 / 4}$. Therefore, we obtain that for $2 \leq p \leq 4$ the radius of the distribution is given by [18]:

$$
R_{p}^{2}=\frac{2}{p} \sqrt{1-\xi}
$$

being a disk for $p=2$, of the form $\sim\left(R_{3}^{2}-\lambda^{2}\right)^{-1 / 2}$ for $p=3$, and for $p \geq 4$ a spherical shell of radius $R_{p}=\left(\frac{1-\xi}{4}\right)^{1 / 4}$.

Note that at $\xi=1$ the radius of the distribution collapses to zero. This suggests that the model is unstable in this regime, which is consistent with the analysis of the previous section. 
There is another interesting set of values of the parameter $\xi$ for which the ground state wave function (3.17) is exact for any $N[19,20]$. Indeed, the order $1 / N$ term that we ignored when substituting equation (3.17) into equation (3.16) is given by:

$$
\frac{(6-3 p)(\sqrt{1-\xi}-1)+(7-4 p) \xi}{6 N} \frac{1}{N^{2}} \sum_{i \neq j} \frac{1}{\left(\vec{\lambda}_{i}-\vec{\lambda}_{j}\right)^{2}} .
$$

Imposing that the coefficient in front of the double sum vanishes we obtain the following set of values of the parameter $\xi$ :

$$
\xi=0, \quad \text { or } \quad \xi=\frac{3(5 p-8)(p-2)}{(4 p-7)^{2}},
$$

for which the ground wave function is exact for any $N$. The case $\xi=0$ was analysed in ref. [10], while for the other case we have to check that it is consistent with the stability condition $\xi<1$, obtained above. Indeed one can check that for $p \geq 2$ the second solution is in the range $0 \leq \xi<\frac{15}{16}$, which is consistent.

\section{Conclusions}

We have studied a quantum mechanical system comprised of a set of $p$ commuting matrices whose Hamiltonian has $\mathrm{SO}(p)$ symmetry. Our starting point was the curved configuration space for commuting matrices. We found that this space is flat only for the one matrix case, and in general has a scalar curvature (A.10) which is singular and negative for all $p>1$ when pairs of eigenvalues approach one another.

As a byproduct we caculated the scalar curvature, of a generic flag manifold, where the different radii squashing the standard flag, $\Delta_{i j}$, are given by the rotationally invariant differences of eigenvalues $\Delta_{i j}^{2}=\left(\vec{\lambda}_{i}-\vec{\lambda}_{j}\right)^{2}$. The flag curvature is given by the relatively simple expression

$$
\mathcal{R}_{x}=N \sum_{i \neq j} \frac{1}{\Delta_{i j}^{2}}-\frac{1}{2} \sum_{i \neq j \neq k} \frac{\Delta_{j k}^{2}}{\Delta_{i j}^{2} \Delta_{i k}^{2}},
$$

see (A.12).

The negative curvature comes from the extrinsic curvature contribution, $\mathcal{K}_{\lambda}$, to the total curvature and is given in (A.12).

We, unfortunately, were unable to solve for the ground state wavefunction for a generic potential. However, we established that with the curvature coefficient $\xi \geq 1$ the system has no finite energy ground state and so, irrespective of the potential the eigenvalues collapse. When the coupling $\xi$ is less than one we treated the case of a quadratic potential and found the ground state energy $E=\frac{1}{2} \sqrt{1-\xi}+O(1 / N)$ and the wavefunction was Gaussian with an eigenvalue repulsion coefficient given by $\Delta_{i j}^{\sqrt{1-\xi}}$.

One advantage of considering generic $\xi$ is that it allows us to make contact with the literature for higher dimensional Calogero-Sutherland-Marchioro type models, since the Hamiltonian for our system (3.16) with quadratic potential is of this type (see equations (7) of [19] and (3) of [20]). 
In a fashion analogous to $p=1$, where both Fermionic and Bosonic quantisations were possible, for $p=2$ an alternative quantisation is also possible. Though we have not pursued this line of investigation, in the case of $p=2$ it is interesting to note that one could have taken the analytic square root of the eigenvalue difference by going to complex coordinates and make contact with anyonic systems [21]. The Hamiltonian in this case can be mapped into a multi-anyon system as in eq. (3) of [22].

The novel rôle played by $p=4$ is that once the number of commuting matrices is four or more the eigenvalues form infinitesimally thin shells. We establish this when the potential is Gaussian but we suspect that it may be a more general result. The study of this question warrants another study and we hope to return to this question in the future.

\section{A Scalar curvature of the space of commuting hermitian matrices}

In this appendix we derive the expression for the scalar curvature of the space of commuting hermitian matrices (3.9). Let us consider the induced metric on this space given in equation (3.4), which we duplicate bellow:

$$
d s^{2}=\sum_{i} d \vec{\lambda}_{i}^{2}+2 \sum_{i>j}\left(\vec{\lambda}_{i}-\vec{\lambda}_{j}\right)^{2} \theta_{i j} \bar{\theta}_{i j}
$$

A key observation is that the dependence of the metric on the directions of the flag manifold is entirely in terms of the left invariant Maurer-Cartan forms $\theta_{i j}$ (more precisely the off diagonal ones for $i \neq j$ ). This implies that the homogeneous structure of the flag is preserved and at fixed eigenvalues (fixed $\vec{\lambda}_{i}$ ) all points on the flag are equivalent in the sense that the vicinity of all points looks the same. This implies that the scalar curvature should not depend on the flag directions and can be calculated locally at any point of the flag. The most convenient choice is to calculate the scalar curvature at the origin of the flag manifold. Since the curvature involves up to second derivatives of the metric we conclude that it is sufficient to know the explicit parametrisation of the metric up to second order near the origin of the flag.

Let us describe our choice of parametrisation. The parametrisation of a general element of $\mathrm{U}(N)$ near unity is given by $U=e^{i x}$, where $x$ is an $N \times N$ hermitian matrix with $N^{2}$ independent degrees of freedom. The flag manifold is obtained by factoring with respect to all diagonal unitary matrices and hence is described by $N(N-1)$ independent parameters $\omega^{a}$. The flag can then be embedded in the $\mathrm{U}(N)$ manifold by the parametrisation $x(\omega)$. In general the closed form of such a parametrisation is rather complex, however it can be constructed easily locally - in the vicinity of the unity element of $\mathrm{U}(N)$. Indeed, to third order in $x$ we have:

$$
U=\hat{1}+i x-\frac{1}{2} x \cdot x-\frac{i}{6} x \cdot x \cdot x+O\left(x^{4}\right)
$$

and using the definition $\theta=U^{\dagger} d U$ for the off diagonal Maurer-Cartan forms we obtain:

$$
\theta_{i j}=i d x_{i j}-\frac{1}{2}\{x, d x\}_{i j}-\frac{i}{6}\left\{x^{2}, d x\right\}_{i j}-\frac{i}{6}(x . d x \cdot x)_{i j}+O\left(x^{3}\right) .
$$

One can see that at the origin $(x=0)$ the off diagonal Muarer-Cartan forms are given by $i d x_{i j}$ and are hence parametrised by the off diagonal elements of $x$, exactly $N(N-$ 
1) real degrees of freedom. Therefore there is always a sufficiently small vicinity of the unit element, where the off diagonal Muarer-Cartan forms can be parametrised by the off diagonal elements of the hermitian matrix $x$ and the embedding of the flag in these coordinates is simply given by $x_{i i}=0$ and $x(\omega)_{i j}=\omega_{i j}$ for $i \neq j$. Our strategy in calculating the scalar curvature will be to treat the off diagonal elements $x_{i j}$ as complex coordinates. For the components of the metric to second order in $x_{i j}$ we obtain:

$$
\begin{aligned}
G_{\lambda_{i}^{\mu}, \lambda_{j}^{\nu}}= & \delta_{i j} \delta_{\mu \nu}, \\
G_{x_{i j}, x_{m l}}= & \Delta_{i j}^{2} \delta_{i l} \delta_{j m}+\frac{i}{2}\left(x_{l i} \delta_{j m}-x_{j m} \delta_{i l}\right)\left(\Delta_{i j}^{2}-\Delta_{m l}^{2}\right)+ \\
& +\frac{1}{12}\left[\left(3 \Delta_{i s}^{2}-2 \Delta_{i j}^{2}-2 \Delta_{i m}^{2}\right) x_{j s} x_{s m} \delta_{i l}+\left(3 \Delta_{m s}^{2}-2 \Delta_{m l}^{2}-2 \Delta_{i m}^{2}\right) x_{l s} x_{s i} \delta_{m j}\right]+ \\
& +\frac{1}{12}\left(4 \Delta_{i j}^{2}-3 \Delta_{j l}^{2}-3 \Delta_{i m}^{2}+4 \Delta_{m l}^{2}\right) x_{l i} x_{j m}, \\
G_{\lambda_{i}^{\mu}, x_{l m}}= & G_{x_{l m}, \lambda_{i}^{\mu}}=0,
\end{aligned}
$$

where $\Delta_{i j}^{2} \equiv\left(\vec{\lambda}_{i}-\vec{\lambda}_{j}\right)^{2}$. The calculation of the components of the associated Levi-Chevita connection is straightforward, the non-zero components (up to first order in $x$ ) are:

$$
\begin{aligned}
\Gamma_{x_{k s}, x_{q p}}^{\lambda_{i}^{\mu}=} & -\left(\delta_{i k}-\delta_{i s}\right) \delta_{k p} \delta_{q s} \Delta_{k s}^{\mu}+\frac{i}{2}\left(x_{s q} \delta_{k p}-x_{p k} \delta_{q s}\right)\left(\left(\delta_{i k}-\delta_{i s}\right) \Delta_{k s}^{\mu}+\left(\delta_{i q}-\delta_{i p}\right) \Delta_{p q}^{\mu}\right), \\
\Gamma_{\lambda_{i}^{\mu}, x_{q p}}^{x_{k s}}= & \left(\delta_{i q}-\delta_{i p}\right) \delta_{k q} \delta_{p s} \frac{\Delta_{k s}^{\mu}}{\Delta_{k s}^{2}}+\frac{i}{2 \Delta_{k s}^{2}}\left(\delta_{i p} \delta_{p s} x_{k q} \Delta_{k q}^{\mu}+\delta_{i k} \delta_{k q} x_{p s} \Delta_{p s}^{\mu}\right)- \\
& -\frac{i}{2 \Delta_{k s}^{2}}\left(\delta_{i p}-\delta_{i s}\right) \delta_{k q} x_{p s} \Delta_{i k}^{\mu}-\frac{i}{2 \Delta_{k s}^{2}}\left(\delta_{i k}-\delta_{i q}\right) \delta_{p s} x_{k q} \Delta_{i p}^{\mu}, \\
\Gamma_{x_{k s}, x_{p q}}^{x_{l m}}= & \frac{i}{2 \Delta_{l m}^{2}}\left(\delta_{k q} \delta_{p l} \delta_{s m}-\delta_{l k} \delta_{m q} \delta_{s p}\right)\left(\Delta_{k s}^{2}-\Delta_{p q}^{2}\right)+\frac{1}{12} \delta_{k q}\left(x_{s m} \delta_{l p}+x_{l p} \delta_{m s}\right)+ \\
& +\frac{1}{4} \delta_{p s}\left(x_{q m} \delta_{k l}+x_{l k} \delta_{m q}\right)\left(\frac{1}{3}+\frac{\Delta_{k p}^{2}}{\Delta_{k m}^{2}}-\frac{\Delta_{l p}^{2}}{\Delta_{l q}^{2}}-\frac{\Delta_{m p}^{2}}{\Delta_{k m}^{2}}+\frac{\Delta_{p q}^{2}}{\Delta_{l q}^{2}}\right)+ \\
& +\frac{1}{4} \delta_{k q}\left(x_{s m} \delta_{l p}-x_{l p} \delta_{m s}\right)\left(\frac{\Delta_{k l}^{2}}{\Delta_{l m}^{2}}-\frac{\Delta_{k m}^{2}}{\Delta_{l m}^{2}}+\frac{\Delta_{k s}^{2}}{\Delta_{s p}^{2}}-\frac{\Delta_{p q}^{2}}{\Delta_{s p}^{2}}\right)+ \\
& +\frac{1}{4}\left(x_{s p} \delta_{k l} \delta_{m q}+x_{q p} \delta_{l p} \delta_{m s}\right)\left(-\frac{2}{3}+\frac{\Delta_{k p}^{2}}{\Delta_{l m}^{2}}-\frac{\Delta_{k s}^{2}}{\Delta_{l m}^{2}}-\frac{\Delta_{p q}^{2}}{\Delta_{l m}^{2}}+\frac{\Delta_{s q}^{2}}{\Delta_{l m}^{2}}\right),
\end{aligned}
$$

where $\Delta_{i j}^{\mu} \equiv \lambda_{i}^{\mu}-\lambda_{j}^{\mu}$ and again $\Delta_{i j}^{2}=\left(\vec{\lambda}_{i}-\vec{\lambda}_{j}\right)^{2}$.

The next step is to use the standard formula for the Riemman curvatute tensor:

$$
\mathcal{R}_{\sigma \mu \nu}^{\rho}=\partial_{\mu} \Gamma_{\nu \sigma}^{\rho}-\partial_{\nu} \Gamma_{\mu \sigma}^{\rho}+\Gamma_{\mu \lambda}^{\rho} \Gamma_{\nu \sigma}^{\lambda}-\Gamma_{\nu \lambda}^{\rho} \Gamma_{\mu \sigma}^{\lambda}
$$

and the definition for the scalar curvature $\mathcal{R}=g^{\sigma \nu} \mathcal{R}_{\sigma \lambda \nu}^{\lambda}$, to arrive at the expression (3.9):

$$
\begin{aligned}
\mathcal{R} & =-(4 p+3 N-10) \sum_{i \neq j} \frac{1}{\left(\vec{\lambda}_{i}-\vec{\lambda}_{j}\right)^{2}}+\frac{3}{2} \sum_{i \neq j \neq k} \frac{\left(\vec{\lambda}_{j}-\vec{\lambda}_{k}\right)^{2}}{\left(\vec{\lambda}_{i}-\vec{\lambda}_{j}\right)^{2}\left(\vec{\lambda}_{i}-\vec{\lambda}_{k}\right)^{2}} \\
& =-4(p-1) \sum_{i \neq j} \frac{1}{\left(\vec{\lambda}_{i}-\vec{\lambda}_{j}\right)^{2}}-3 \sum_{i \neq j \neq k} \frac{\left(\vec{\lambda}_{i}-\vec{\lambda}_{j}\right)}{\left(\vec{\lambda}_{i}-\vec{\lambda}_{j}\right)^{2}} \cdot \frac{\left(\vec{\lambda}_{i}-\vec{\lambda}_{k}\right)}{\left(\vec{\lambda}_{i}-\vec{\lambda}_{k}\right)^{2}},
\end{aligned}
$$


where we have used the relation:

$$
\sum_{i \neq j \neq k} \frac{\left(\vec{\lambda}_{j}-\vec{\lambda}_{k}\right)^{2}}{\left(\vec{\lambda}_{i}-\vec{\lambda}_{j}\right)^{2}\left(\vec{\lambda}_{i}-\vec{\lambda}_{k}\right)^{2}}=2(N-2) \sum_{i \neq j} \frac{1}{\left(\vec{\lambda}_{i}-\vec{\lambda}_{j}\right)^{2}}-2 \sum_{i \neq j \neq k} \frac{\left(\vec{\lambda}_{i}-\vec{\lambda}_{j}\right)}{\left(\vec{\lambda}_{i}-\vec{\lambda}_{j}\right)^{2}} \cdot \frac{\left(\vec{\lambda}_{i}-\vec{\lambda}_{k}\right)}{\left(\vec{\lambda}_{i}-\vec{\lambda}_{k}\right)^{2}} .
$$

We can present another useful result. Note that if we keep the eigenvalues fixed, the expression for the connection involving only components along the flag directions, equation (A.8), represents the connection of a flag manifold with constant radii $\left|\vec{\Delta}_{i j}\right|=\sqrt{\Delta_{i j}^{2}}$. The result in equation (A.10) can be split into contributions from the flag directions $\mathcal{R}_{x}$ and contribution from the mixed terms $\mathcal{K}_{\lambda}$, the two terms read:

$$
\begin{aligned}
& \mathcal{R}_{x}=N \sum_{i \neq j} \frac{1}{\Delta_{i j}^{2}}-\frac{1}{2} \sum_{i \neq j \neq k} \frac{\Delta_{j k}^{2}}{\Delta_{i j}^{2} \Delta_{i k}^{2}}, \\
& \mathcal{K}_{\lambda}=-(4 p-2) \sum_{i \neq j} \frac{1}{\Delta_{i j}^{2}}-4 \sum_{i \neq j \neq k} \frac{\vec{\Delta}_{i j} \cdot \vec{\Delta}_{i k}}{\Delta_{i j}^{2} \Delta_{i k}^{2}} .
\end{aligned}
$$

Equation (A.12) is our expression for the curvature of a flag manifold with radii $\left|\vec{\Delta}_{i j}\right|=\sqrt{\Delta_{i j}^{2}}$.

Open Access. This article is distributed under the terms of the Creative Commons Attribution License (CC-BY 4.0), which permits any use, distribution and reproduction in any medium, provided the original author(s) and source are credited.

\section{References}

[1] E. Brézin, C. Itzykson, G. Parisi and J.B. Zuber, Planar Diagrams, Commun. Math. Phys. 59 (1978) 35 [inSPIRE].

[2] Y. Shimamune, On the Phase Structure of Large-N Matrix Models and Gauge Models, Phys. Lett. B 108 (1982) 407 [inSPIRE].

[3] T. Banks, W. Fischler, S.H. Shenker and L. Susskind, $M$ theory as a matrix model: A Conjecture, Phys. Rev. D 55 (1997) 5112 [hep-th/9610043] [INSPIRE].

[4] P.K. Townsend, The eleven-dimensional supermembrane revisited, Phys. Lett. B 350 (1995) 184 [hep-th/9501068] [INSPIRE].

[5] D.E. Berenstein, J.M. Maldacena and H.S. Nastase, Strings in flat space and pp waves from $\mathcal{N}=4$ Super Yang Mills, AIP Conf. Proc. 646 (2003) 3 [hep-th/0202021] [INSPIRE].

[6] O. Aharony, O. Bergman and D.L. Jafferis, Fractional M2-branes, JHEP 11 (2008) 043 [arXiv: 0807.4924] [INSPIRE].

[7] S. Kovacs, Y. Sato and H. Shimada, Membranes from monopole operators in ABJM theory: Large angular momentum and $M$-theoretic $A d S_{4} / C F T_{3}$, Prog. Theor. Exp. Phys. 2014 (2014) 093B01 [arXiv:1310.0016] [InSPIRE].

[8] J. Hoppe, Quantum Theory Of A Massless Relativistic Surface And A Two Dimensional Bound State Problem, Ph.D. Thesis, Massachusetts Institute of Technology (1982).

[9] B. de Wit, J. Hoppe and H. Nicolai, On the Quantum Mechanics of Supermembranes, Nucl. Phys. B 305 (1988) 545 [inSPIRE]. 
[10] D. Berenstein, Large-N BPS states and emergent quantum gravity, JHEP 01 (2006) 125 [hep-th/0507203] [INSPIRE].

[11] B.S. DeWitt, Dynamical theory in curved spaces. 1. A Review of the classical and quantum action principles, Rev. Mod. Phys. 29 (1957) 377 [InSPIRE].

[12] K.S. Cheng, Quantization of a general dynamical system by Feynman's path integration formulation, J. Math. Phys. 13 (1972) 1723 [INSPIRE].

[13] H. Kleinert, Quantum Dynamics in Spaces With Curvature and Torsion, Mod. Phys. Lett. A 4 (1989) 2329 [INSPIRE].

[14] L.D. Landau and E.M. Lifshitz, Quantum Mechanics. Volume 3 of A Course of Theoretical Physics, Pergamon Press (1965).

[15] D. Berenstein, D.H. Correa and S.E. Vazquez, All loop BMN state energies from matrices, JHEP 02 (2006) 048 [hep-th/0509015] [INSPIRE].

[16] O. Aharony and S.A. Hartnoll, A Phase transition in commuting Gaussian multi-matrix models, arXiv:0706.2861 [INSPIRE].

[17] D.E. Berenstein, M. Hanada and S.A. Hartnoll, Multi-matrix models and emergent geometry, JHEP 02 (2009) 010 [arXiv:0805.4658] [inSPIRE].

[18] V.G. Filev and D. O'Connor, On the Phase Structure of Commuting Matrix Models, JHEP 08 (2014) 003 [arXiv: 1402.2476] [INSPIRE].

[19] A. Khare and K. Ray, A Quantum many body problem in two-dimensions: Ground state, Phys. Lett. A 230 (1997) 139 [hep-th/9609025] [INSPIRE].

[20] A. Khare, Exact ground state of several $N$ body problems with an $N$ body potential, J. Math. Phys. 40 (1999) 2640 [cond-mat/9712133] [INSPIRE].

[21] F. Wilczek, Quantum Mechanics of Fractional Spin Particles, Phys. Rev. Lett. 49 (1982) 957 [INSPIRE].

[22] G. Date, M. Krishna and M.V.N. Murthy, Asymptotic analysis and spectrum of three anyons, Int. J. Mod. Phys. A 9 (1994) 2545 [cond-mat/9301021] [INSPIRE]. 